\title{
Chikungunya in the united states
}

\begin{abstract}
Chikungunya is characterized by severe and often enduring febrile arthralgia, which is rarely fatal. The clinical symptoms include febrile poly-arthralgia, and small joints are frequently affected. The general symptomatology is most often headache, asthenia, myalgia and chills, combined with joint swelling, rash, buccal bleeding and mouth ulcer; chronic arthralgia is a frequent complication of acute disease.

The capability of Aedes albopictus to effectively transmit the Asian genotype of the chikungunya virus that was recently found in the Caribbean could facilitate local transmission and spread into the United States towards areas where this invasive mosquito is widespread. Locally acquired infections have been confirmed in the Caribbean, where North American travelers frequently visit. The three major destinations of passengers departing the regions of the Caribbean are the US, France, and the Netherlands Antilles.

Seven months after the disease was detected in the Caribbean Islands, the first autochthonous transmission of infection was reported on July 17, 2014 in Florida in a male patient who had not recently visited any foreign country. The arrival of Chikungunya in the US underscores the risks posed by this and other exotic pathogens. It is not established what course the disease will take now in the US; however, CDC officials believe CHIK will behave like dengue virus, where imported cases have produced only isolated local transmission and have not created epidemic outbreaks.
\end{abstract}

Keywords: Chikungunya virus, Arthralgia, Asian genotype, Outbreak, Globalization, Alphavirus, Arthropod-borne diseases, Aedes aegypti, Aedes albopictus, Arthropod-borne diseases
Volume I Issue 3 - 2014

\author{
Humberto H Lara, Victor H Sepulveda de \\ Leon, Lucia Mureyko, Liliana Ixtepan Turrent \\ Departamento de Ciencias Basicas, Universidad de Monterrey, \\ Mexico
}

Correspondence: Humberto H. Lara, Universidad de Monterrey, Avenida Ignacio Morones Prieto 4500 Poniente Jesús M. Garza 66238; San Pedro Garza García, Nuevo Leo, Mexico, Tel 0I-8I-82I5-1000 ext. 2136 Email d.lara.v@gmail.com

Received: August 08, 2014 | Published: August 22, 2014
Abbreviations: CHIK, Chikungunya Fever; CHIKV, Chikungunya Virus; Aedes, Ae.; ECSA, East-Central-South African; RNA, Ribonucleic Acid; DEN, Dengue

\section{Introduction}

The term chikungunya in the Kimakonde language in Tanzania means "that which bends up" and refers to the contorted posture adopted by aching infected patients. It is a mosquito-borne disease, induced by a virus from the family Togaviridae, genus Alphavirus, and characterized by a severe and often enduring febrile arthralgia that is sporadically fatal. Chikungunya fever or CHIK exists in enzootic nonhuman primate cycles in Africa but occasionally emerges into urban, human cycles to cause major epidemics.

The most common presenting clinical symptoms are febrile polyarthralgia, bilateral in $53.7 \%$ of cases, and small joints were more frequently affected (hands $79.6 \%$, wrists $72.2 \%$, ankles $68.5 \%$, knees $61.1 \%$, lumbar $46.3 \%$, shoulders $48.1 \%$, feet $42.6 \%$, cervix $38.9 \%$ and elbows $25.9 \%$ ); general symptoms are most often headache, asthenia, myalgia and chills. ${ }^{2}$ Less frequent clinical symptoms are joint swellings, rash, buccal bleeding and mouth ulcer. Female patients had a more than one-fold higher relative risk for the symptoms such as rash, vomiting, buccal bleeding and mouth ulcer. ${ }^{3}$ A 36-month prospective longitudinal study demonstrated that chronic arthralgia is a frequent complication of acute CHIK disease and suggested that it results from local rather than systemic inflammation. ${ }^{4}$

The transmission cycle is from humans to mosquitoes, with no intervening amplification; the Chikungunya virus (CHIKV) is able to rapidly disseminate, infecting extensive numbers of individuals to cause major epidemics. These epidemics have been documented in Africa, the Middle East, Europe, India, and Southeast Asia. The mosquitoes, Aedes aegypti and Aedes Albopictus, are the vectors for the CHIKV. A recent single mutation, A226E in the envelope protein 1 (E1) of CHIKV, allows Aedes (Ae.) albopictus as well as Ae. aegypti mosquitoes to transmit CHIKV. ${ }^{5}$ The proven capability of Ae. Albopictus to transmit Asian CHIKV could facilitate the local transmission in the US and South America in areas where Ae. albopictus is common but Ae. aegypti is absent. Entomologic studies have shown that Ae. albopictus can also be found in North America, where it thrives, and Ae. aegypti mosquitoes are disseminated around the tropical and subtropical regions, being a region at risk, where the CHIKV can spread any of its three viral genotypes. ${ }^{6}$

CHIKV is an enveloped virus with a single-stranded, positivesense RNA genome. The $12 \mathrm{~Kb}$ viral genome begins with a UTR at the 5'-terminus, with the following two-thirds of the genome encoding the non-structural proteins (nsP1-4) and the remaining onethird encoding the structural proteins (Capsid-E3-E2-6K-E1) and a 3'-terminal poly-A-tail. ${ }^{7}$ The non-structural proteins are processed into four nsPs and are responsible for different roles in virus replication. nsP1 is important in viral RNA synthesis initiation and viral RNA capping. nsP2 possesses protease and RNA helicase activities. ${ }^{8} \mathrm{nsP} 3$ is composed of three domains, and this viral protein is required for the formation and localization of replication complexes. nsP4 possesses RNA-dependent RNA polymerase (RdRp) activity that is important for replication and synthesis of the viral genome. ${ }^{9}$

\section{CHIKV genotypes}

There are three CHIKV genotypes, the East-Central-South African (ECSA), West African, and Asian; the ECSA genotype has been correlated with the recent epidemics in the Indian Ocean region, and the Asian genotype has been associated with the current outbreaks in the Pacific region. A correlation to the epidemic in the La Reunion outbreak was a single-base mutation in 1 strain of the ECSA genotype, enhancing the replication of the virus in the mosquito Ae. Albopictus. ${ }^{10}$ The Asian genotype was found in the recent detection of the CHIKV in a Caribbean island (Saint Martin) in October 2013. ${ }^{10}$ 


\section{CHIKV in Africa and Asia}

CHIKV was first identified in Tanzania in 1952 and was later found to be widely distributed and to cause sporadic, mostly small outbreaks in Africa (Democratic Republic of Congo, Nigeria, Angola, Uganda, Guinea, Malawi, Central African Republic, Burundi and South Africa) and Asia (Burma, Thailand, Cambodia, Vietnam, India, Sri Lanka, Timor, Indonesia and the Philippines) during the 1960's and 1990's. Then, in June 2004, an epidemic occurred on Lamu Island, Kenya and spread to Comoros, La Reunion, Indian Ocean islands, causing approximately 500,000 cases. ${ }^{11}$ Subsequently, from 2006-2009, CHIKV became even more present in India and produced more than 1.5 million cases. Consequently, CHIKV has now been recognized as a major emerging, epidemic-prone pathogen.

\section{CHIKV in Sierra Leone}

In Sierra Leone in 1972, cases of CHIKV infection were detected Recently two developments made a reemergence appear imminent. The first of these developments was the outbreak of CHIKV in neighboring Guinea and in Senegal, and the second was the observation that Aedes spp. are common in Sierra Leone, as evidenced by the number of yellow fever cases in this country. On July 7, 2012, researchers at the Mercy Hospital Research Laboratory in Bo, Sierra Leone initiated a 1-year infectious disease surveillance program to identify which pathogens are causing febrile illness in their city. By January 10, 2013, 400 (42.9\%) of 932 febrile patients were detected as positive for the CHIKV by lateral flow immunoassay. ${ }^{12}$

\section{CHIKV in the Americas (Caribbean)}

The first evidence for autochthonous cases of CHIKV infection in the Western hemisphere was reported in December 2013 on the island Saint Martin. ${ }^{13}$ Locally acquired infections have been confirmed in 17 countries or territories in the Caribbean or South America (Guyana, French Guiana, Anguilla, Antigua and Barbuda, British Virgin Islands, Dominica, Dominican Republic, Guadeloupe, Haiti, Martinique, Puerto Rico, Saint Barthélemy, Saint Kitts and Nevis, Saint Lucia, Saint Martin, Saint Vincent and the Grenadines,), where a large number of North American travelers habitually vacation; these travellers could potentially introduce the disease into the US. ${ }^{14}$ In addition, warmer climates in North America will translate into more mosquitoes and more opportunity for autochthonous outbreaks. ${ }^{10}$

\section{The source of the disease}

Between the months of October and December 2012, five countries were the main origin of $84.4 \%$ of all of the international air passengers leaving the CHIK-endemic areas of the world with a final destination in the Caribbean region. These countries comprise South Africa (23.4\%), India (21.6\%), China (13.8\%), Philippines (13.7\%) and the French territory of La Réunion (11.9\%). ${ }^{13}$

The three major destinations of the passengers departing the areas of the Caribbean are the US 52.2\%, France $14.5 \%$, and the Netherlands Antilles, not including Saint Martin 3.3\%. By comparison, ten cities represented the final destination of $49 \%$ of all international travelers. These cities included New York (13.8\% of the global total), Paris (11.7\%), Miami (7.8\%), San Juan, Puerto Rico (3.9\%), Curacao (2.4\%), Fort Lauderdale (2.2\%), Madrid (2.0\%), Boston (1.9\%), Toronto (1.7\%), and Caracas (1.4\%).

\section{Transmission vectors}

Ae. Aegypti: Also known as the yellow fever mosquito, Ae. aegypti has been present in the US since the beginning of colonization and is alleged to have been brought from the African continent by
European pioneers through commerce and transportation. This vector has the capabilities to flourish and replicate in urban settings, lives in close relationship with people and has more of a predilection for feeding on humans than any other host. The female mosquito feeds to nourish her eggs and is more dynamic during daylight. ${ }^{15}$ This arthropod can thrive in the American East from the Gulf Coast to the Middle Atlantic states and beyond towards the populous New York city. The eggs can resist drought for extended periods of time, which boosts the dissemination of the arthropods, depending on the mechanism of transportation. The Ae. aegypti mosquito is active in the Northern United States during the summertime and active year round in the South, benefiting from the warm climate. ${ }^{13}$

Ae. Albopictus: Commonly called the Asian tiger mosquito (Skuse), Ae. Albopictus is considered to be the most invasive mosquito species and is of vast interest from a public health perspective; this mosquito species inhabits the Southeast United States, the Caribbean region and the Gulf coast of Mexico. ${ }^{16}$ The Asian tiger mosquito prevails in urban areas and flourishes in 36 states in the US. ${ }^{17}$ The mosquito adapts to both urban and rural surroundings, has a flight radius of 400 to 600 meters and is an intruding daylight biter. The Asian tiger mosquito's eggs are resistant to drought and cold weather, consequently enhancing the invasive spread of this pest. Over the last 30 years, this arthropod has been spread rapidly by human travel and commerce from its native tropical forests of Asia to every continent except Antarctica. ${ }^{18}$ Broad regions of the American continent are vastly crowded with Ae. aegypti and Ae. albopictus mosquitoes, and millions of dengue (DEN) infections are recorded each year. ${ }^{19}$

\section{Risk of dissemination throughout the US}

Seven months after the arthropod-borne CHIKV was identified in the Caribbean Islands, the first autochthonous transmission of infection of CHIK has been reported in the continental U.S. The case was communicated on July 17, 2014 and was diagnosed in Florida in a male patient who had not recently toured any foreign country.

Since 2006, the US has averaged 28 imported infections of CHIK annually in passengers returning from countries with the disease. Altogether during 2014, 243 imported cases have been recorded in 31 states and two territories. This year, Puerto Rico and the US Virgin Islands reported 121 and two cases of locally acquired CHIK to the CDC, respectively. "The arrival of chikungunya virus, first in the tropical Americas and now in the US, underscores the risks posed by this and other exotic pathogens," said Roger Nasci, chief of CDC's Arboviral Diseases Branch.

\section{Treatment}

No licensed vaccine or specific medication is currently available. Treatment is palliative and consists of rest, hydration, and the use of analgesics and antipyretics. ${ }^{21}$ Most patients' symptoms improve within 1 week. Joint pain can persist for months. ${ }^{4}$ One method to diagnose the disease is by measuring the levels of $\operatorname{IgM}$ antibodies against the CHIKV, which are usually measurable by immunochromatographic methods within a few days after infection and remain elevated for approximately 3 to 4 months. ${ }^{22}$

\section{Prevention}

The best way to prevent CHIK is to avoid Aedes mosquito bites by using the following precautions: use air conditioning or window and door screens when indoors, use insect repellents, and wear long sleeves and pants when outdoors. Persons infected with CHIKV should be protected from mosquito exposure during the first week of illness to prevent the further spread of the virus to the community. ${ }^{23}$ 
Passengers returning from countries where the CHIKV is present and those residing in states where the CHIKV has been detected in the continental US should pursue medical attention if they experience CHIK symptoms. Health care providers in areas with reported cases should be on the alert for probable cases. Patients infected with CHIKV should protect themselves by applying insect repellents, using air conditioning or window and door screens to exclude the mosquitoes, wearing long pants and long sleeved shirts, and discharging standing water in the outdoors. Protecting oneself and others from mosquito bites during the first few days of illness can help to prevent other mosquitoes from becoming infected and reduce the risk of further spread.

It is still not known what course CHIK will take now in the US. CDC officials believe that CHIK will behave like dengue virus in the US, where imported cases have resulted in sporadic local transmission but have not caused widespread outbreaks. Local transmission has been reported in 23 countries in the hemisphere prior to the US case.

\section{Discussion}

There are favorable conditions for the local transmission of CHIK in the US; the main reasons are: a) the capacity of Ae. Albopictus to transmit the Asian genotype of CHIKV, which is prevalent in the Caribbean and which could facilitate the local transmission and spread of the disease in the US in areas where the mosquito is known to be common; b) the three major destinations of passengers departing the infected areas of the Caribbean are the US, France, and the Netherlands Antilles; c) the first autochthonous transmission of infection has been reported on July 17, 2014 in Florida in a male patient who had not recently toured any foreign country; d) CDC officials believe CHIK will behave like dengue virus in the US, where imported cases have resulted in sporadic local transmission but have not caused widespread outbreaks; and e) the population has no immunity to this pathogen, which is new in the US.

\section{Conclusion}

The first case of local transmission of CHIK has been reported in Florida, where there are conditions that support local transmission and dissemination of the virus. Prevention is the key element in combating the disease, because there is no treatment or vaccine against this painful disease. More research to discover vaccines and antivirals against this virus and to contain the Aedes mosquito are urgently needed.

\section{Acknowledgment}

None.

\section{Conflict of interest}

None.

\section{References}

1. Van Bortel W, Dorleans F, Rosine J et al. Chikungunya outbreak in the Caribbean region, December 2013 to march 2014, and the significance for Europe. Euro Surveill. 2014;19(13):20759.

2. Thiberville SD, Moyen N, Dupuis-Maguiraga L et al. Chikungunya fever: epidemiology, clinical syndrome, pathogenesis and therapy. Antiviral Res. 2013;99(3):345-370.

3. Razmy AM Clinical features of chikungunya infection in Sri Lanka. Asian Pac J Trop Dis. 2014;4(2):2014;131-34.
4. Schilte C, Staikowsky F, Staikovsky F et al. Chikungunya virus-associated long-term arthralgia: a 36-month prospective longitudinal study. PLoS Negl Trop Dis. 2013;7(3):e2137.

5. Tsetsarkin KA, Chen R, Sherman MB et al. Chikungunya virus: evolution and genetic determinants of emergence. Curr Opin Virol. 2011;1(4):310-317

6. Dogan ADA, Bunes K, Skarphedinsson S The tropical disease chikungunya fever has come to europe. Ugeskr Laeger. 2013;175(24):1716-1719.

7. Chen KC, Kam Y-W, Lin RTP et al. Comparative analysis of the genome sequences and replication profiles of chikungunya virus isolates within the east, central and South African (ecsa) lineage. Virol J doi: 10.1186/1743-422X-10-169. 2013

8. Caglioti C, Lalle E, Castilletti $\mathrm{C}$ et al. Chikungunya virus infection: an overview. New Microbiol. 2013;36(3):211-227.

9. Butt AM, Nasrullah I, Tong Y Genome-wide analysis of codon usage and influencing factors in chikungunya viruses. PLoS One. 2014;9(3):e90905.

10. Leparc-Goffart I, Nougairede A, Cassadou S et al. Chikungunya in the americas. Lancet 383(9916): 514.

11. Pialoux G, Gauzere BA, Jaureguiberry S et al. Chikungunya, an epidemic arbovirosis. Lancet Infect Dis 2007;7(5):319-27.

12. Ansumana R, Jacobsen KH, Leski TA et al. Reemergence of chikungunya virus in Bo, Sierra Leone. Emerg Infect Dis. 2013;19(7):1108-1110.

13. Khan K, Bogoch I, Brownstein JS et al. Assessing the origin of and potential for international spread of chikungunya virus from the Caribbean. PLoS Curr. 2014;6:14.

14. Fischer M, Staples JE, Arboviral Diseases Branch, National Center for Emerging and Zoonotic Infectious Diseases, CDC Notes from the field: chikungunya virus spreads in the Americas - Caribbean and South America, 2013-2014. MMWR Morb Mortal Wkly Rep. 2014;63(22):500-501.

15. Saifur RGM, Dieng H, Hassan AA et al. Changing domesticity of Aedes aegypti in northern peninsular Malaysia: reproductive consequences and potential epidemiological implications. PLoS One. 2012;7(2):e30919.

16. Burt FJ, Rolph MS, Rulli NE et al. Chikungunya: a re-emerging virus. Lancet. 2012;379(9816):662-671.

17. Rochlin I, Ninivaggi DV, Hutchinson ML et al. Climate change and range expansion of the Asian tiger mosquito (Aedes albopictus) in Northeastern USA: implications for public health practitioners. PLoS One 2012;8(4):e60874.

18. Zhong $\mathrm{D}$, Lo $\mathrm{E}, \mathrm{Hu} \mathrm{R}$ et al. Genetic analysis of invasive Aedes albopictus populations in los Angeles county, California and its potential public health impact. PLoS One. 2013;8(7):e68586.

19. Vega-Rua A, Zouache K, Girod R et al. High level of vector competence of Aedes aegypti and Aedes albopictus from ten American countries as a crucial factor in the spread of chikungunya virus. J Virol 2014;88(11):6294-6306.

20. http://www.cdc.gov/media/releases/2014/p0717-chikungunya.html

21. Parashar D, Cherian S Antiviral perspectives for chikungunya virus. Biomed Res Int doi.org/10.1155/2014/631642. 2014

22. Kosasih H, Widjaja S, Surya E et al. Evaluation of two IgM rapid immunochromatographic tests during circulation of Asian lineage chikungunya virus. Southeast Asian J Trop Med Public Health. 2012;43(1):55-61.

23. Majra JP, Acharya D Impact of knowledge and practices on prevention of chikungunya in an epidemic area in India. Ann Trop Med Public Health. 2011;4(1):3-6. 